EUROPEAN UNIVERSITY INSTITUTE, FLORENCE

DEPARTMENT OF LAW

EUI Working Paper LAW No. 2004/6

Le dispositif Dublin 2

ou les tribulations de la politique communautaire d'asile

SÉGOLÈNE BARBOU DES PLACES 
All rights reserved.

No part of this paper may be reproduced in any form without permission of the author(s)

(C) 2004 Ségolène Barbou des Places

Published in Italy in March 2004

European University Institute

Badia Fiesolana

I - 50016 San Domenico (FI)

Italy 


\section{LE DISPOSITIF DUBLIN 2 \\ OU LES TRIBULATIONS DE LA POLITIQUE COMMUNAUTAIRE D’ ASILE}

\section{Ségolène Barbou des PlaceS}

Marie Curie Fellow, Institut Universitaire Européen de Florence, Département Droit Maître de Conférences, Université Nancy 2 
La Convention de Dublin relative à la détermination de l'Etat responsable de l'examen d'une demande d'asile présentée dans l'un des Etats membres des Communautés européennes, adoptée en 1990, a mal fonctionné et le traité d'Amsterdam a instauré la base juridique de son remplacement par un règlement communautaire. Dans ses travaux préparatoires, la Commission proposait une réforme radicale du système qui rénovait l'esprit du système Dublin et pouvait en assurer l'efficacité.

Mais la «réforme » annoncée n'a pas eu lieu. Cet article s'efforce de montrer que les deux règlements 343/2003 du 18 février 2003 et 1560/2003 du 2 septembre 2003 n'innovent pas. Le Conseil a choisi le statu quo et l'attente. La logique d'attribution de la responsabilité de 1990 est reconduite et la caractéristique du règlement du 18 février 2003 semble être une certaine myopie à l'égard des migrations d'asile. En fait, les institutions communautaires oscillent entre une approche méfiante des stratégies de déplacement et de choix de l'Etat de destination, l'utopie d'un possible contrôle de ces déplacements et de ces choix, et l'illusion que Dublin 2 pourrait être efficace. Cet article montre les limites d'un réglement encastré dans le processus d'harmonisation, et dont l'efficacité est compromise.

Keywords: Dublin Asylum Convention, Asylum Policy, Harmonisation, European Law. 


\section{INTRODUCTION}

Lors du Conseil européen de Tampere de 1999, les Etats membres de 1'Union européenne sont convenus de mettre en place un régime d'asile européen commun et comportant, à court terme, une méthode claire et opérationnelle pour déterminer l'Etat membre responsable de l'examen d'une demande d'asile. C'est à cette fin qu'a été adopté le règlement 343/2003 du Conseil du 18 février 2003 établissant les critères et mécanismes de détermination de l'Etat membre responsable de l'examen d'une demande d'asile présentée dans l'un des Etats membres par un ressortissant d'un pays tiers ${ }^{1}$. Le dispositif, désormais dénommé « Dublin 2 », fut rendu opérationnel par l'adoption du règlement d'application le 2 septembre $2003^{2}$.

Les deux règlements de février et septembre 2003 remplacent un instrument de droit international, la Convention de Dublin ${ }^{3}$, qui fut adoptée en 1990 pour tenter de répondre à trois problèmes rencontrés par les Etats en matière d'asile. Le premier problème est celui des migrations dites secondaires des demandeurs d'asile dans la Communauté européenne. Il s'agit de l'hypothèse où un demandeur d'asile entre dans la CE par un Etat, dans lequel il ne dépose pas de demande d'asile, puis voyage jusqu'à un autre Etat où il demande l'asile car il considère que ce dernier pays lui offre une protection plus favorable (protection contre le refoulement, droits économiques, sociaux etc.). Les Etats ont décidé d'empêcher ces migrations secondaires car elles entraînent une répartition très inégale des demandeurs d'asile entre eux ainsi que des charges financières très disparates. Le deuxième problème est celui des demandes d'asile multiples qui surchargent les administrations nationales chargées du traitement des demandes d'asile. Enfin, le troisième problème fut désigné par l'appellation «réfugiés sur orbite ». Il s'agit de la situation de personnes ayant déposé une demande d'asile dans plusieurs Etats quand aucun Etat ne se considère responsable du traitement de leur demande.

Pour résoudre ces trois problèmes, la Convention de Dublin a consacré le principe de l'unicité de l'Etat compétent pour examiner une demande d'asile. Ce principe signifie qu'un seul Etat partie à la Convention, désigné sur la base de

\footnotetext{
${ }^{1} \mathrm{JO}, \mathrm{L} 50 / 1$, du 25.2.2003

2 Règlement 1560/2003 de la Commission du 2 septembre 2003 portant modalités d'application du règlement 343/2003 du Conseil établissant les critères et mécanismes de détermination de l'Etat responsable de l'examen d'une demande d'asile présentée dans l'un des Etats membres par un ressortissant d'un pays tiers, J.O. L122, 5.9.2003, p. 3-23.

${ }^{3}$ JOCE C 254, 19.08.1997
} 
six critères ${ }^{4}$, est responsable de l'examen du dossier d'un demandeur d'asile. Le système préserve les ressources des Etats car si une personne dépose plusieurs demandes d'asile, un seul Etat examine le dossier. Le principe garantit également que chaque demande d'asile présentée en Europe sera examinée, et répond ainsi au problème des réfugiés sur orbite. Une fois déterminée, la responsabilité entraîne pour l'Etat membre concerné l'obligation d'admettre le demandeur d'asile sur son territoire, de traiter sa demande et le cas échéant, de le réadmettre s'il s'est rendu sans autorisation dans un autre Etat membre ${ }^{5}$.

Malgré les décisions du comité 18 (comité chargé par l'article 18 de la bonne application de la Convention), l'application de la Convention, entrée en vigueur en $1997^{6}$, fut rapidement problématique. Ainsi, le mécanisme de désignation de l'Etat responsable du traitement de la demande d'asile s'est avéré inefficace car contourné par les demandeurs d'asile préférant souvent une situation irrégulière et précaire à l'application des critères Dublin $^{7}$. De nombreuses stratégies d'évitement du dispositif Dublin ont été relevées ${ }^{8}$. Dans de nombreux cas, le

${ }^{4}$ Le premier critère est celui du regroupement familial : est désigné compétent 1'Etat dans lequel certains membres de la famille du demandeur d'asile ont obtenu le statut de réfugié. Par application du deuxième critère, est désigné compétent l'Etat qui a délivré un permis de séjour en cours de validité. Le troisième critère désigne l'Etat ayant délivré un visa en cours de validité. Par application du quatrième critère, est compétent l'Etat par lequel le demandeur d'asile est entré illégalement en Europe. Le cinquième critère vise l'entrée légale sur le territoire national. Enfin, au cas où aucun des critères précédents n'est applicable, sera désigné responsable l'Etat dans lequel le demandeur d'asile aura déposé sa première demande d'asile.

${ }^{5}$ La Convention de Dublin organise la procédure de prise en charge d'un demandeur d'asile (hypothèse où le demandeur d'asile est installé dans un Etat membre qui ne se considère pas compétent et demande à un autre Etat partie de prendre en charge le demandeur d'asile) et de la reprise en charge (hypothèse où le demandeur d'asile a déposé une demande d'asile dans un Etat compétent et a ensuite voyagé vers un autre Etat membre.)

${ }^{6}$ La Convention est entrée en vigueur le 1er septembre 1997. Elle liait alors douze Etats membres. Elle est entrée en vigueur en Suède et en Autriche en octobre 1997 et en Finlande en 1998.

${ }^{7}$ Voir Liébaut, F., The Dublin Convention, Study on its implementation in the 15 Member States of the EU, www.flyghtning.dk/publikationer. Voir aussi les contributions de Karsten Kloth et Gloria Bodelon Alonson in Claudia Faria (ed.) The Dublin Convention on Asylum. Between Reality and Aspirations, Institut Européen d'Administration Publique, 2001.

${ }^{8}$ Si par exemple l'Etat de prédilection des demandeurs d'asile se trouve à la fin de leur route en Europe, ils tentent par tous moyens, y compris clandestinement, d'arriver à destination. S'ils réussissent à accéder à l'Etat voulu, ils détruisent parfois leurs documents de voyage et font une fausse description de la route empruntée pour éviter d'être rattachés à l'Etat de transit. Si l'Etat finalement désigné compétent n'est pas celui où ils veulent demander l'asile, il tentent d'échapper à la procédure de transfert. Pour cela, ils choisissent parfois de disparaitre et choisissent la clandestinité. Parfois ils retirent leur demande d'asile, ce qui ne produit d'ailleurs aucun effet juridique. Ou bien, pour éviter tous risques, ils décident de ne pas déposer une demande en vue d'obtenir le statut de réfugié - qui déclenche l'application des critères Dublin - mais déposent une demande de protection sur un autre fondement que la 
système Dublin a également porté atteinte à l'unité familiale des demandeurs d'asile et réfugiés, et ce malgré l'existence de la clause dérogatoire dite humanitaire permettant à un Etat non compétent d'examiner une demande d'asile pour des raisons humanitaires fondées notamment sur des motifs familiaux (article 3-4). En effet, la Convention n'a jamais été conçue comme une norme conférant des droits subjectifs aux demandeurs d'asile et l'application de cette clause est laissée à la discrétion des Etats.

Enfin, du point de vue des Etats, le système n'est pas non plus apparu performant: le système s'est avéré coûteux pour de faibles résultats ${ }^{9}$. L'application des critères Dublin a été minée par des problèmes de communication entre les Etats et des difficultés de preuve ${ }^{10}$. La lenteur de la détermination de l'Etat responsable a rendu précaire la situation des demandeurs d'asile et les transferts prévus par la Convention ont concerné un nombre très faible de demandeurs d'asile ${ }^{11}$. Sans doute parce qu'il repose sur une fiction - en ce qu'il prétend pouvoir répartir, sur la base de critères préétablis, des personnes entre 15 Etats, sans prendre en compte ni l'intérêt des Etats ni celui des personnes ${ }^{12}$, ni les différences de législation existant entre les Etats ${ }^{13}-1 \mathrm{e}$ dispositif d'origine est un échec.

Convention de Genève, i.e. un statut dit subsidiaire ou complémentaire. Dans ce dernier cas la Convention de Dublin n'est pas applicable mais la protection accordée est amoindrie en comparaison de celle prévue au titre de la qualité de réfugié conventionnel.

${ }^{9}$ Voir le bilan présenté dans le document de travail "Evaluation de la Convention de Dublin", SEC(2001)756 final du 13.06.2001. Voir aussi les chiffres commentés par le Comité économique et social qui relève que le mécanisme laborieux mis en place n'est appliqué que pour une très faible proportion des demandes d'asile et moins de $1,7 \%$ des demandeurs d'asile sont finalement transférés vers un autre Etat que celui dans lequel ils ont déposé leur demande, in Opinion du Comité économique et social sur la proposition de règlement du Conseil établissant les critères et mécanismes de détermination de l'Etat membre responsable d'une demande d'asile présentée dans l'un des Etats membres par un ressortissant d'Etat tiers du 20 mars 2002, CES 352/2002.

${ }^{10}$ Voir Friedrich Löper, The Dublin Convention Provisions on Time Limits and the Exchange of Information: Which Are the Provisions and What Are the Problems?, in Claudia Faria (ed.) The Dublin Convention on Asylum. Between Reality and Aspirations, Institut Européen d'Administration Publique, 2001, pp. 27-35

${ }^{11}$ Idem et voir "Réexamen de la Convention de Dublin : élaboration d'une législation communautaire permettant de déterminer quel Etat membre est responsable de l'examen d'une demande d'asile présentée dans l'un des Etats membres", SEC(2000)522 final du 21.03.2000.

${ }^{12} \mathrm{~A}$ juste titre le parlement britannique considère que l'échec de la Convention de Dublin tient à ce que les Etats ont présumé, à tort, que les demandeurs d'asile et les Etats de première admission pourraient s'accomoder de procédures qui fonctionnent à leur détriment, in Nineteenth Report of the UK Parliament, 19 March 2002, by the Select Commitee appointed to consider Union documents and other matters relating to the European Union, www.parliament-the-stationery-office.co.uk 
C'est le traité d'Amsterdam qui a instauré la base juridique pour l'adoption d'une norme communautaire établissant les critères et mécanismes de détermination de l'Etat responsable de l'examen d'une demande d'asile présentée dans l'un des Etats membres (article 63-1-a). Il fut en effet prévu en 1997 de remplacer la Convention de Dublin par une norme directement et uniformément applicable sur le territoire communautaire ${ }^{14}$. La Commission a préparé le futur règlement par une série d'études préparatoires (la Convention de Dublin fut évaluée en 2000 et $2001^{15}$ ) et une proposition de règlement fut déposée en octobre $2001^{16}$. Soucieuse d'assurer l'efficacité du dispositif Dublin sans pour autant modifier la logique de 1990, la Commission a déposé une proposition « offensive ». Elle préconisait diverses dispositions qui limitaient de façon drastique l'autonomie des demandeurs d'asile, empêchaient les demandeurs d'asile de contourner le mécanisme de détermination de l'Etat responsable et imposaient aux Etats un contrôle très strict des flux migratoires. Mais cette proposition était une impasse : elle portait atteinte aux obligations internationales des Etats et empiétait sur la compétence des Etats. De manière générale, l'approche préconisée par la Commission contredisait l'intention exprimée par les Etats lors du Conseil européen de Tampere de mettre en place un régime d'asile européen fondé sur l'application intégrale et globale de la Convention de Genève ${ }^{17}$.

Le règlement 343/2003 est une version épurée de la proposition de la Commission. Il met en place un dispositif qui doit être examiné avec attention.

${ }^{13}$ On a parfois eu recours à l'expression "protection loterie" car les demandeurs d'asile, selon l'Etat membre auquel ils seront rattachés, pourront espérer des niveaux de protection très disparates, y compris contre le refoulement.

${ }^{14}$ On ne peut pas négliger cependant les difficultés d'application qui devraient découler de la non application du règlement Dublin au Danemark, (celui ci conformément au protocole sur la position du Danemark annexé au traité sur l'Union européenne et au traité instituant la Communauté européenne, ne participant pas à l'adoption du règlement et n'étant pas lié par lui.)

${ }^{15}$ La discussion a été initiée par un document de travail des services de la Commission "Réexamen de la Convention de Dublin : élaboration d'une législation communautaire permettant de déterminer quel Etat membre est responsable de l'examen d'une demande d'asile présentée dans l'un des Etats membres", SEC(2000)522 final du 21.03.2000. Puis la Commission a élaboré une "Evaluation de la Convention de Dublin", SEC(2001)756 final du 13.06.2001.

${ }^{16}$ Proposition de règlement du Conseil établissant les critères et mécanismes de détermination de l'Etat membre responsable de l'examen d'une demande d'asile présentée dans l'un des Etats membres par un ressortissant d'un pays tiers, COM(2001)0447 final, JO, C 304 du 30.10.2001, p. 192.

${ }^{17}$ La mention dans ce texte de la Convention de Genève doit évidemment être comprise comme une référence à la Convention du 28 juillet 1951 relative au statut des réfugiés, modifiée par le protocole de New York du 31 janvier 1967. 
Le système Dublin a rarement les honneurs des commentaires doctrinaux car c'est un dispositif technique qui n'affecte pas directement la substance des droits nationaux de l'asile. Pourtant, un examen attentif de la réforme de la Convention de 1990 révèle les contradictions et insuffisances de l'approche des Etats face au phénomène migratoire particulier qu'est l'asile. Le système Dublin témoigne des atermoiements des institutions communautaires et des Etats face à une réalité qu'il est difficile d'appréhender au niveau européen et de régir par le droit. C'est pourquoi le présent article propose une analyse du système Dublin 2, en s'attachant à sa genèse et à ses possibles conséquences. Il s'intéresse en particulier aux options politiques privilégiées par le Conseil, lorsque elles diffèrent de la proposition de la Commission. Les choix opérés révèlent les limites d'un mécanisme qui bien souvent s'avère(ra) inadapté aux réalités européennes de l'asile. Dublin 2 en effet n'est qu'un léger toilettage du dispositif de 1990: c'est un dispositif de raison (I) et de saison (II).

\section{PARTIE I. UN DISPOSITIF DE RAISON : L'ABANDON DES DISPOSITIONS OFFENSIVES PRECONISEES PAR LA COMMISSION}

Le règlement Dublin doit être analysé par comparaison avec la proposition déposée par la Commission le 30 octobre 2001. Cette proposition poursuivait un objectif principal : assurer l'effectivité du dispositif Dublin. A cette fin, les dispositions préconisées révélaient une approche offensive des migrations d'asile. Mais le Conseil a opté pour une attitude plus modérée et surtout conforme à la légalité (A). Il reconduit les le lien conceptuel entre le système Dublin et l'exigence de contrôle des frontières mais renonçe à transformer le système Dublin en un instrument de lutte contre l'immigration clandestine (B).

\section{Section 1. RETOUR A LA LEGALITE}

Pour assurer l'efficacité du dispositif Dublin, la Commission proposait deux dispositions empêchant les demandeurs d'asile de contourner ou éviter l'application des critères de désignation de l'Etat responsable. La première est le projet d'article 4-3 qui prévoyait que la substitution d'une demande de protection sur un autre fondement que la Convention de Genève à une demande d'asile dûment introduite «ne fait pas obstacle à la poursuite de la procédure de détermination de l'Etat responsable ». L'objectif de la Commission était d'empêcher un demandeur d'asile de retirer sa demande de reconnaissance de la qualité de réfugié et de la remplacer par une demande de protection subsidiaire dans le seul but de faire échec à l'application des critères Dublin et de pouvoir 
ainsi échapper au transfert. La deuxième disposition est du même type : au titre du projet d'article 20-2, la décision de transfert d'un demandeur d'asile vers l'Etat responsable de l'examen de sa demande était susceptible de recours juridictionnel, mais le recours ne pouvait pas avoir d'effet suspensif. La Commission préconisait la suppression de l'effet suspensif de l'appel pour éviter un recours considéré comme dilatoire et empêcher les demandeurs d'asile de profiter de la période de suspension pour disparaître.

Dans la version finale du règlement, ces deux articles ont simplement disparu. Plusieurs raisons expliquent l'abandon de ces dispositions, l'une d'elles étant la volonté du Conseil de réaliser l'objectif réaffirmé à Tampere d'une politique communautaire d'asile conforme à la Convention de Genève et aux obligations internationales des Etats.

\section{$\S 1$. La mise en conformité avec la légalité internationale}

L'application de l'article 20-2 était problématique, dans la mesure où elle risquait de conduire les Etats à une situation de violation de leurs obligations au regard de la Convention de Genève et de la Convention européenne des droits de l'homme. Pour justifier cette disposition, la Commission avait en effet dû recourir à la fiction de l'équivalence des niveaux de protection en Europe. Ainsi elle considérait que «un transfert vers un autre Etat membre n'étant pas de nature à causer à la personne concernée un préjudice grave et difficilement irréparable, il n'est pas nécessaire que l'exécution du transfert soit suspendue dans l'attente du résultat de la procédure contentieuse $»^{18}$. De même elle estimait que la proposition de maintien de la procédure Dublin en cas de modification de la demande de protection " ne lèse en rien les personnes concernées puisque les Etats membres ont tous souscrit aux mêmes obligations en ce qui concerne le respect des droits fondamentaux, la protection contre la torture et les peines et traitements inhumains ou dégradants et la protection contre le refoulement. ${ }^{19}$

Cette fiction de l'équivalence des protections ${ }^{20}$ n'est pourtant pas acceptable. Elle a été considérée comme un cas grave de myopie ${ }^{21}$ susceptible d'entraîner

\footnotetext{
${ }^{18}$ Proposition de règlement du Conseil établissant les critères et mécanismes de détermination de l'Etat membre responsable de l'examen d'une demande d'asile présentée dans l'un des Etats membres par un ressortissant d'un pays tiers, COM(2001)0447 final, JO, C 304 du 30.10.2001, p. 192, commentaire de l'article 20-2

${ }^{19}$ Proposition de règlement du Conseil établissant les critères et mécanismes de détermination de l'Etat membre responsable de l'examen d'une demande d'asile présentée dans l'un des Etats membres par un ressortissant d'un pays tiers, COM(2001)0447 final, JO, C 304 du 30.10.2001, p. 192, commentaire de l'article 4-3.

${ }^{20}$ Un autre projet d'article reposait sur la fiction de l'équivalence des protections nationales: c'est le projet d'article 24-1c. Il permettait aux Etats membres d'établir entre eux des arrangements administratifs et notamment d'instaurer « un mécanisme de rationalisation des
} 
une violation du droit international. On peut en effet se représenter le risque que représente la suppression de l'effet suspensif, pour un demandeur d'asile persécuté par des agents non étatiques, qui voit désigné pour l'examen de sa demande un Etat membre considérant que ce type de persécution n'entre pas dans le champ d'application de la Convention de Genève, et risque le refoulement dans son pays d'origine. Un tel transfert, qui équivaudrait à un refoulement indirect constitue une violation de la Convention de Genève et de la Convention européenne des droits de 1 'homme ${ }^{22}$. Le projet d'article 20-2 qui interdit aux juges nationaux de suspendre l'application du transfert quand le transfert est préjudiciable au demandeur d'asile, fut donc logiquement critiqué par l'ensemble des observateurs ${ }^{23}$.

transferts permettant (...) de procéder au transfert des seuls cas dont un Etat membre demeure débiteur vis-à-vis de l'autre Etat membre une fois qu'il a été renoncé aux prises en charge qui s'annulent mutuellement ». La Commission voulait ainsi régir les hypothèses où les Etats connaissent un volume important de prises et reprises en charge qui s'effectuent dans les deux sens, aboutissant à ce qu'elle appelle «une sorte de chassé-croisé de demandeurs d'asile faisant l'objet de transferts consentis ou sous escorte ». La Commission suggérait donc « de renoncer de part et d'autre à mettre en œuvre un nombre égal de transferts afin de procéder seulement au transfert, dans un seul sens, d'un nombre limité de personnes, désignés sur la base de critères appropriés, correspondant à la différence entre les transferts acceptés de chaque côté. » Ce mécanisme reposait sur le postulat que, pour un demandeur d'asile, il y a équivalence entre transfert et non-transfert, ce qui revient à admettre l'équivalence des protection offertes par tous les Etats.

${ }^{21}$ Gregor Noll, Denying difference: The Dublin Successor Regulation, in Asylum in Europe : Strategies, Problems and Solutions, Report from the Nordic Refugee Seminar, Lund, 28-29 August 2001, in édition du Raoul Wallenberg Institute, Lund, 2002.

${ }^{22}$ La Cour européenne des droits de l'homme a rappelé dans l'affaire TI contre Royaume Uni (7 mars 2000) que les Etats ne sauraient échapper à leur responsabilité individuelle à l'aide d'une notion de responsabilité collective, que ce soit au titre de la Convention de Dublin ou de toute autre Convention. Un transfert qui risque de conduire, même indirectement, à un traitement inhumain ou dégradant, entraînera responsabilité de l'Etat.

${ }^{23}$ Voir en particulier Hemmes Mattjes, A balance between Fairness and Efficiency? The Directive on International Protection and The Dublin Regulation, European Journal of Migration and Law, 4-2002, pp. 159-192 et Gregor Noll, in Asylum in Europe : Strategies, Problems and Solutions, Report from the Nordic Refugee Seminar, Lund, 28-29 August 2001, in édition du Raoul Wallenberg Institute, Lund 2002. Pour les ONG, on peut se reporter aux analyses critiques faites par ILPA (Immigration Law Practicionners Association), ILPA's Scoreboard on the proposal for a Council Regulation establishing the criteria and mechanisms for determining the Member State responsible for examining an asylum application lodged in one of the Member State by a third country national, disponible sur www.ilpa.org.uk; ou encore Amnesty International's comments on the Commission's proposal for a Council Regulation establishing the criteria and mechanisms for determining the Member State responsible for examining an asylum application lodged in one of the Member State by a third country national, www.amnesty.org. Voir enfin Comments from the European Council on Refugees and Exiles on the Proposal for a Council Regulation establishing the criteria and mechanisms for determining the Member State responsible for examining an asylum application lodged in one of the Member States by a third country national, www.ecre.org. 
Le règlement évite ces griefs d'illégalité et n'interdit plus aux juges de prononcer l'effet suspensif de l'appel contre le transfert. Il ne prévoit cependant pas un effet suspensif systématique. La voie retenue est une solution médiane puisque le règlement admet, dans ses articles 19 final et 20, que la décision « n'a pas d'effet suspensif sur l'exécution du transfert, sauf lorsque les tribunaux ou les instances compétentes le décident, au cas par cas, si la législation nationale le permet $\gg$.

L'abandon des projets d'articles 4-3 et 20-2 trouve également sa source dans le souci de la conformité au traité $\mathrm{CE}$, en particulier à la lettre de la base juridique du règlement Dublin, l'article 63-1-a TCE.

\section{§2- Le respect de la légalité communautaire}

Les deux projets d'articles étaient critiquables car non conformes à l'article 631-a TCE, disposition sur laquelle est fondé le règlement Dublin. La proposition d'article 20-2, tout d'abord, était contestable. Au titre des articles 20-1 et 21-1 de la proposition de règlement, une décision de transfert ne peut en effet être prise qu'après détermination de l'Etat responsable du traitement de la demande. L'application de l'article 20-2 supprimant l'effet suspensif d'un transfert ne pourrait donc avoir lieu que après la désignation de l'Etat compétent. Dès lors la base juridique appropriée pour l'article 20-2 ne pouvait être l'article 63-1-a TCE qui vise exclusivement les «critères et mécanismes permettant de déterminer l'Etat responsable de l'examen d'une demande d'asile ». La seule base juridique envisageable pour une disposition telle que le projet d'article 20-2 était donc l'article 63-1-d TCE qui prévoit l'adoption de normes minimales concernant la procédure d'octroi ou de retrait du statut de réfugié dans les Etats membres.

Or, ce même article 63-1-d TCE admet l'adoption ou le maintien par les Etats de mesures nationales plus favorables. En revanche le projet d'article 20-2 empêchait tout Etat d'avoir recours à une disposition plus favorable puisque le juge national ne pouvait pas suspendre l'application du transfert. Cette rigidité réalisait les craintes de Gregor Noll de voir le règlement Dublin, instrument de droit communautaire uniformément appliqué sur le territoire européen, supprimer tout espace pour les pratiques nationales plus généreuses ${ }^{24}$. L'empiétement sur la compétence de l'article 63-1-d était d'autant plus problématique que sur ce fondement, est en discussion la proposition de

\footnotetext{
${ }^{24}$ Gregor Noll, in Asylum in Europe : Strategies, Problems and Solutions, Report from the Nordic Refugee Seminar, Lund, 28-29 August 2001, édition du Raoul Wallenberg Institute, Lund 2002. p. 11
} 
directive procédure ${ }^{25}$ dont l'article 33-1 prévoit que l'appel a un effet suspensif. La proposition d'article 20-2 était donc en contradiction à la fois avec le traité $\mathrm{CE}$ et avec la proposition de directive procédure. En renonçant au projet d'article 20-2, le Conseil semble donc admettre que le règlement Dublin n'est qu'un des six moyens prévus par l'article 63 TCE pour réaliser la politique européenne d'asile. Il doit contribuer à la réalisation de la politique d'asile et complète pour cela les directives d'harmonisation prévues ou en cours d'adoption, mais il ne saurait se substituer à ces mesures ni y porter atteinte.

C'est probablement le même raisonnement qui sous-tend le retrait du projet d'article 4-3. L'adoption du texte sur la continuation du mécanisme Dublin en cas de retrait de la demande de statut de réfugié devait permettre, selon la Commission, «de mettre à égalité les Etats membres où existent des procédures distinctes pour accéder à des formes de protection complémentaires avec les Etats membres où, par l'effet d'une procédure unique, la détermination de la forme de protection appropriée n'est pas laissée au choix du demandeur ». La Commission s'efforçait donc de neutraliser les effets des différences de législation. Elle considérait que lorsque, dans les Etats membres où cela est rendu possible par l'existence de procédures distinctes, un demandeur d'asile modifie en cours de procédure la nature de sa demande de protection, en particulier dans le but d'échapper à l'application du dispositif de détermination de l'Etat responsable, ce choix du demandeur d'asile ne devrait, en principe, pas faire obstacle à la continuation de la procédure. En ne permettant plus aux demandeurs d'asile de contourner le dispositif Dublin par l'utilisation de règles spécifiques à certains Etats, la Commission voulait donc assurer une application uniforme du système Dublin sur tout le territoire communautaire.

Mais cette approche était critiquable car, en s'efforçant d'unifier l'application du dispositif Dublin et de gommer les différences entre les législations nationales, le projet d'article 4-3 sortait du champ d'application de l'article 63-1-a. En effet, seules les personnes qui demandent la reconnaissance du statut de réfugié sont visées par l'article 63-1-a TCE. Dès lors, une personne qui retire sa demande de statut de réfugié doit pouvoir échapper au champ d'application du règlement ${ }^{26}$. Or l'article 4-3 maintenait l'application des critères Dublin dans une hypothèse de retrait de la demande de statut de réfugié et de dépôt d'une demande de protection subsidiaire. L'hypothèse défendue ici est donc que le Conseil

\footnotetext{
${ }^{25}$ Proposition modifiée de directive du Conseil relative à des normes minimales concernant la procédure d'octroi et de retrait du statut de réfugié dans les États membres, COM/2002/0326 final.

${ }^{26}$ Voir sur ce point la position de ILPA's Scoreboard on the proposal for a Council Regulation establishing the criteria and mechanisms for determining the Member State responsible for examining an asylum application lodged in one of the Member State by a third country national, précitée, p. 4.
} 
abandonne le projet d'article 4-3 pour mettre le contenu du règlement Dublin en conformité avec la norme le fondant juridiquement. Ce faisant, le Conseil admet aussi que dans certains Etats, la substitution d'une demande de protection complémentaire à une demande de statut de réfugié permettra d'échapper à l'application des critères Dublin. L'application du système Dublin sera donc susceptible de variations locales, mais cette solution est préférée à l'intérprétation hasardeuse des termes du traité $\mathrm{CE}$ envisagée par la Commission.

En abandonnant les projets d'articles 4-3 et 20-2, le Conseil a donc purgé le règlement Dublin de tout risque d'illégalité et le règlement du 18 février 2003 est plus modéré que la proposition de la Commission. La prudence du Conseil se manifeste également dans le fait que l'équilibre de 1990 entre les critères déterminant l'Etat compétent pour examiner une demande d'asile est reconduit.

\section{SECTION 2- STATU QUO SUR LES CRITERES DE RESPONSABILITE}

Dès l'origine, le mécanisme Dublin a été pensé en relation avec l'objectif de réalisation d'un espace sans frontières internes. Aussi, un lien conceptuel fut-il établi dans la Convention entre l'attribution de la responsabilité du traitement de la demande d'asile et le contrôle des migrations. Malgré les modifications apportées par le règlement, cette logique est préservée (1). Contrairement à la proposition de la Commission, le règlement n'arrime cependant pas le dispositif Dublin à la lutte contre l'immigration clandestine (2).

§1- L'instrumentalisation du dispositif Dublin au service du contrôle des frontières

Une lecture rapide du règlement Dublin peut faire croire à la refonte des critères de détermination de l'Etat compétent car la nouvelle liste des critères de désignation de l'Etat responsable de l'examen de la demande semble être un compromis entre les impératifs des contrôles frontaliers, l'intérêt des Etats, et celui des familles. En particulier, le règlement contient de nouvelles dispositions qui favorisent le regroupement familial et, situation nouvelle, les critères attribuant la responsabilité à l'Etat sur la base du regroupement familial s'appliquent avant les critères relatifs à la responsabilité de l'Etat pour l'entrée et le séjour du demandeur d'asile sur son territoire.

Une analyse plus précise révèle toutefois que Dublin 2 reste, comme son prédécesseur, un instrument au service des contrôles migratoires et n'est pas transformé en un instrument d'asile. Ainsi, la responsabilité est toujours attribuée à l'Etat qui, par un acte positif tel que la délivrance d'un visa ou d'un titre de séjour, a pris la plus grande part dans l'entrée et le séjour d'un 
ressortissant d'Etat tiers dans l'UE ou qui, par une défaillance, a permis l'entrée irrégulière. Au titre du nouvel article 9, sera déclaré compétent l'Etat qui a délivré un titre de séjour ou un visa en cours de validité. Au titre de l'article 10, est responsable l'Etat dans lequel le demandeur d'asile est entré irrégulièrement en venant d'un Etat tiers ou, quand les circonstances de l'entrée ne peuvent être établies avec clarté, l'Etat dans lequel le demandeur d'asile a séjourné de manière continue au moins 5 mois avant l'introduction de sa demande (10-2). Selon l'article 11, est responsable l'Etat d'entrée régulière ou de dépôt de la demande d'asile quand l'entrée sur le territoire européen n'est pas soumise à une condition de visa ${ }^{27}$. L'article 11 est justifié par le même lien entre responsabilité du traitement de la demande d'asile et responsabilité du contrôle migratoire car comme l'indique la Commission, lorsque le ressortissant de pays tiers est dispensé de visa, “on ne saurait considérer que l'Etat par lequel il est entré dans l'espace commun a été défaillant vis-à-vis de ses partenaires en autorisant l'entrée puisque tout autre Etat membre aurait autorisé l'entrée dans les mêmes conditions". Le principe de désignation de l'Etat responsable de la demande d'asile demeure donc fondé sur l'idée que, dans un espace de libre circulation, chaque Etat membre est "comptable vis-à-vis de tous les autres de son action en matière d'entrée et de séjour des ressortissants des Etats tiers" $" 28$.

Malgré les modifications apportées par le règlement, Dublin 2 n'est donc pas devenu un instrument de politique d'asile. La référence à la Convention de Genève reste minimale. La possibilité de renvoyer un demandeur d'asile vers un Etat tiers sûr n'est pas supprimée ${ }^{29}$. De plus, aucune garantie n'est prévue pour faire échec à un transfert vers un Etat désigné compétent si ce transfert est préjudiciable au demandeur d'asile. Le règlement traite donc avant tout du contrôle de la circulation des demandeurs d'asile dans une Europe aux frontières internes abolies.

Or l'utilisation d'un mécanisme de répartition de la compétence à des fins de contrôle migratoires a montré ses limites. Le lien entre responsabilité de l'examen de la demande et contrôle des entrées en Europe incite les Etats à refouler illégalement les demandeurs d'asile à leurs frontières terrestres et maritimes. Il conduit les Etats à exporter les mesures d'endiguement en

${ }^{27}$ Lorsqu'un ressortissant d'un pays tiers entre sur le territoire d'un Etat membre dans lequel il est exempté de l'obligation de visa, l'examen de sa demande d'asile incombe à cet Etat membre. Mais si ce ressortissant d'Etat tiers introduit sa demande dans un autre Etat membre dans lequel il est exempté de l'obligation de visa, c'est ce dernier Etat qui est responsable de la demande d'asile.

${ }^{28}$ Proposition de règlement, commentaire de l'article 9.

${ }^{29}$ L'article 3-3 dispose que "tout Etat membre conserve la possibilité, en application de son droit national, d'envoyer un demandeur d'asile vers un Etat tiers, dans le respect des dispositions de la Convention de Genève" 
envoyant des officiers d'immigration dans les pays de transit ou d'origine ${ }^{30}$. On connaît également la tendance à faire peser la responsabilité des contrôles sur les transporteurs par le biais des différentes législations prévoyant leur responsabilité ${ }^{31}$. Ces différentes mesures constituent des barrières qui empêchent les demandeurs d'asile d'accéder à la protection, et contredisent l'affirmation du Conseil européen de Tampere selon laquelle l'Union et les Etats membres accordent un respect absolu au droit de demander l'asile et s'engagent à offrir des garanties à tous ceux qui cherchent protection ou accès à l'Union européenne.

Peut être le Conseil, cependant, n'était-il pas insensible à ces arguments car il a renoncé aux dispositions sanctionnant les Etats pour une défaillance dans le traitement de l'immigration clandestine.

\section{$\S 2$-Le refus des critères relatifs à l'immigration clandestine}

La proposition de la Commission imposaient aux Etats une stricte surveillance de l'immigration. Un projet d'article 12 prévoyait en particulier que «L'Etat membre qui a toléré sciemment la présence irrégulière du ressortissant d'un pays tiers sur son territoire pendant une période supérieure à deux mois est responsable de l'examen de la demande d'asile ». Un article 13-1 établissait que «S'il peut être démontré que le ressortissant d'un pays tiers a séjourné six mois ou plus en situation irrégulière sur le territoire d'un Etat membre, cet Etat est responsable de l'examen de la demande d'asile».

La justification de ces deux articles est intéressante. L'article 12 était motivé par l'idée que l'Etat qui a toléré la situation de séjour irrégulier a, "par son inertie, favorisé les projets du ressortissant d'un pays tiers qui a pu attendre sur son territoire une occasion favorable pour se rendre irrégulièrement dans un autre Etat membre » afin d'y déclarer son intention de solliciter la reconnaissance de la qualité de réfugié. Pour la Commission, la menace d'un éloignement aurait au contraire conduit la personne concernée, si elle estime qu'un retour dans son pays d'origine l'exposerait à la persécution, à introduire une demande d'asile dont l'existence aurait permis de mettre en œuvre la procédure de détermination de l'Etat responsable en vertu du présent règlement. On imagine aisément que ces propos ont pu être dictés par l'expérience franco-britannique du centre de

\footnotetext{
${ }^{30}$ Voir Danièle Joly, A New Asylum regime in Europe, in F. Nicholson, P. Twomey (sous dir.)Refugee, Rights and Realities. Evolving International Concepts and Regimes, Cambridge University Press, 1999, pp. 336-356. Voir également Karen Landgren, Deflecting International Protection by Treaty: Bilateral and Multilateral Accords on Extradition, reAdmission and the Inadmisibility of Asylum Request, UNHCR's New Issues in Refugee Research, 1999, Working Paper $\mathrm{N}^{\circ} 10$, www. unhcr.ch

31 Antonio Cruz, Shifting responsibility, carrier's liability in the membre states of the European Union and North America, Trentham Book, 1995.
} 
Sangatte, même si celle ci n'est pas mentionnée explicitement. L'article 13 pour sa part était justifié par l'idée que l'Etat qui n'a pas détecté la présence irrégulière d'un ressortissant d'Etat tiers a été défaillant dans la mise en œuvre des objectifs de contrôle de l'immigration clandestine communs aux Etats membres et doit en assumer les conséquences vis à vis de ses partenaires.

Pour contraindre les Etats à lutter efficacement contre le séjour irrégulier, la Commission avait donc établi un lien de causalité entre le défaut de contrôle des séjours clandestins et la responsabilité de l'examen de la demande d'asile. Une telle approche est critiquable, tout d'abord, car elle instrumentalise le dispositif Dublin et en fait un outil de la politique de lutte contre l'immigration clandestine en Europe, ce qui n'est pas sa mission. Elle est ensuite préoccupante car elle repose sur l'idée de sanction des Etats défaillants. Dans l'exposé des motifs de l'article 11, il est en effet fait mention de l'idée qu'un Etat doit ou ne doit pas être " pénalisé ». Ce vocabulaire contribue à propager l'idée que l'attribution de la responsabilité de l'examen d'une demande d'asile à un Etat est une sanction, une peine, qui découle d'une action erronée ou d'une omission. Cette terminologie doit inquiéter car elle s'éloigne très fortement de la conception selon laquelle la charge de l'examen d'une demande d'asile est la réponse apportée par un Etat, en vertu de ses obligations internationales, à une personne dont on a de fortes raisons de supposer qu'elle craint une persécution.

Le règlement ne retient pas la logique contestable de la Commission et abandonne les deux projets d'articles 12 et $13^{32}$. On se réjouira tout d'abord de la disparition des termes relatifs à la peine ou sanction : le règlement raisonne en terme de responsabilité, et non en terme de pénalité. Par ailleurs, le retrait des projets d'articles 12 et 13 est judicieux car les dispositions préconisées par la Commission, bien que destinées à assurer l'efficacité du mécanisme Dublin, risquaient d'être contre-productives. On pouvait en premier lieu s'interroger sur la viabilité du mécanisme. Sous le régime Dublin 1, le critère de responsabilité basé sur l'entrée irrégulière n'est en effet quasiment jamais appliqué en raison de difficultés de preuve qu'il rencontre : il est presque impossible de savoir par quel Etat un demandeur d'asile entre dans l'Union européenne. Comment alors démontrer qu'un Etat membre a toléré la présence irrégulière d'un demandeur d'asile pendant deux mois ou qu'un demandeur d'asile a séjourné dans un Etat membre plus de 6 mois ? La Commission attendait probablement une importante contribution d'Eurodac sur ce point, mais il n'est pas certain qu'Eurodac suffise pour prouver un séjour irrégulier dans un Etat ou déterminer sa durée. De surcroît, la logique de sanction retenue n'incitait pas les Etats à révéler la

\footnotetext{
${ }^{32}$ Il supprime également l'article 17-3 qui reposait sur la même logique en prévoyant que s'il peut être démontré que le demandeur d'asile a séjourné au moins 6 mois dans un Etat membre, les obligations imposables à l'Etat chargé de prendre ou reprendre en charge le demandeur d'asile sont transférées à cet Etat membre.
} 
présence de clandestins sur leur territoire. JUSTICE ${ }^{33}$ craignait aussi que les Etats n'aient la tentation de renvoyer les demandeurs d'asile vers d'autres Etats membres au plus vite, i.e. avant que les délais prévus aux articles 12 et 13 n'expirent. Le risque était donc important, si les Etats et les demandeurs d'asile avaient intérêt à la clandestinité, que les articles 12 et 13 soient ineffectifs.

En renonçant à ces articles, le règlement Dublin manifeste le réalisme du Conseil. Il refuse un mécanisme à l'efficacité compromise, qui risquait au surplus d'inciter les Etats à adopter des comportements contraires à l'intérêt collectif. On ne peut qu'adhérer à l'analyse d'Elspeth Guild qui décrivait le projet d'article 12 par les remarques suivantes : "il est difficile d'imaginer un système qui soit mieux conçu pour porter atteinte à l'intégration européenne. La confiance est une des caractéristiques les plus difficiles à établir dans le système européen. Pourtant elle est indispensable à l'intégration communautaire. Certains mécanismes qui renforcent la méfiance et diminuent la confiance entre les Etats sont contraires à l'intégration européenne. La proposition de règlement Dublin entre malheureusement dans cette catégorie" ${ }^{34}$.

Enfin en retirant les projets d'articles 12 et 13, le Conseil évite le développement d'effets pervers. Puisqu'il suffisait d'un séjour irrégulier sur le territoire d'un Etat pour que cet Etat soit déclaré responsable de la demande d'asile, l'article 13 risquait d'être une "incitation » à la clandestinité. Un demandeur d'asile entré par un Etat périphérique de l'Union mais désireux de demander l'asile dans un autre Etat pouvait en effet développer la stratégie suivante: entrer clandestinement (pour échapper à l'application du critère entrée irrégulière ; projet d'article 10), puis voyager jusqu'à l'Etat de prédilection, y rester caché pendant 6 mois puis y demander l'asile. Après 6 mois de séjour irrégulier, le demandeur d'asile voyait désigner l'Etat de son choix compétent pour examiner sa demande ${ }^{35}$.

\footnotetext{
${ }^{33}$ JUSTICE, cité in Nineteenth Report of the UK Parliament, 19 March 2002, by the Select Commitee appointed to consider Union documents and other matters relating to the European Union, www.parliament-the-stationery-office.co.uk

${ }^{34}$ Elspeth Guild, citée in Nineteenth Report of the UK Parliament, 19 March 2002, by the Select Commitee appointed to consider Union documents and other matters relating to the European Union, www.parliament-the-stationery-office.co.uk (traduit par nos soins).

35 Il n'est pas impossible que l'abandon du projet d'article 18-4 soit explicable par la même volonté de restreindre les stratégies des demandeurs d'asile. Cet article organisant la prise en charge disposait que le demandeur d'asile "est informé sans délai, et dans une langue qu'il comprend, de ce qu'une requête aux fins de prise en charge a été adressée à un autre Etat membre et des délais applicables". On ne voit pas comment justifier le retrait de cette disposition, sinon par la volonté d'éviter que les demandeurs d'asile ne profitent du délai entre l'information et la mise en œuvre du transfert pour disparaitre.
} 
En somme, alors que la proposition de règlement s'efforçait de contrôler certaines stratégies développées par les demandeurs d'asile, le règlement est une version beaucoup plus modeste mais purgée de toute illégalité. Le Conseil a choisi de ne pas innover et reste fidèle aux lignes directrices de 1990. Mais parce qu'il n'est justement pas réformé, le dispositif Dublin 2 reste un instrument inadapté aux réalités de l'asile dans l'Union européenne. Il se révèle sous les traits d'un dispositif temporaire, contraint par le processus d'harmonisation des droits d'asile et d'immigration qu'il précède.

\section{PARTIE II. UN DISPOSITIF DE SAISON : EN ATTENDANT DUblin 3 !}

Le préambule du règlement indique que "il convient, à ce stade [de la réalisation d'un régime d'asile européen commun], tout en y apportant les améliorations nécessaires à la lumière de l'expérience, de confirmer les principes sur lesquels se fonde la Convention (...) signée à Dublin ». Ce passage résume le choix du Conseil. Les critères permettant de désigner l'Etat responsable de l'examen de la demande d'asile ne sont pas modifiés en substance et le Conseil confirme la posture défensive et dirigiste de 1990. Le résultat est un dispositif toujours inadapté aux réalités de l'asile en Europe dont l'efficacité est compromise. Ce choix, pourtant, est explicable. Le dispositif Dublin 2 a été conçu dans un contexte très spécifique: il a été modelé en fonction des autres normes communautaires, adoptées ou en préparation, qui mettent en place la politique commune d'immigration et d'asile. Ainsi la réforme du système Dublin, encastrée dans le processus d'harmonisation, est-elle très superficielle $(A)$, ce qui nuira probablement à l'effectivité du dispositif. L'efficacité du système Dublin, qui était une priorité de la Commission, est déléguée par le Conseil qui met ses espoirs dans des mécanismes annexes de contrôle de l'immigration (B).

\section{SECTION 1. UNE REFORME DIFFEREE : UN REGLEMENT ENCASTRE DANS LE PROCESSUS D'HARMONISATION}

L'adoption du règlement Dublin précède l'harmonisation des droits nationaux sur l'asile et l'immigration. Cette position dans le temps n'est pas sans conséquences. On observe que le système Dublin 2 a été conçu en lien étroit avec les propositions de directives régissant l'immigration. Il est subordonné aux normes fondant le régime commun de l'immigration en Europe (1). De même, l'absence d'harmonisation des droits nationaux de l'asile sert à légitimer la non-réforme du système de 1990 (2). 
Le règlement de février 2003 a toiletté les critères servant à désigner 1'Etat responsable du traitement de la demande d'asile. L'apport le plus significatif est la protection accrue du regroupement familial des demandeurs d'asile. Le règlement ajoute ainsi au texte de 1990 un premier critère de compétence, visant à rapprocher un mineur non accompagné auprès d'un membre adulte de sa famille déjà présent dans un Etat membre et susceptible de le prendre en charge $^{36}$. Il attribue ensuite, par son article 8 , la responsabilité de l'examen de la demande d'asile à l'Etat membre qui examine la demande introduite par un membre de sa famille arrivé antérieurement et qui n'a pas encore fait l'objet d'une décision sur le fond, à condition que les intéressés le souhaitent ${ }^{37}$. Par ailleurs, pour éviter qu'une application littérale des critères de responsabilité ne conduise à séparer les membres d'un groupe familial qui ont introduit une demande dans le même Etat membre, l'article 14 prévoit les dérogations à l'application normale des critères afin de maintenir l'unité du groupe dans un seul Etat membre. Quand plusieurs membres d'une famille introduisent une demande d'asile dans un même Etat membre simultanément ou à des dates suffisamment proches et que l'application des critères du règlement conduirait à les séparer, est désigné responsable, soit l'Etat que les critères désignent comme responsable de la prise en charge du plus grand nombre d'entre eux, soit l'Etat que les critères désignent responsable du plus âgé d'entre eux. Enfin l'article 15, relatif à la clause humanitaire, prévoit désormais que tout Etat, même s'il n'est pas responsable en application des critères Dublin, peut rapprocher les membres d'une même famille ainsi que d'autres parents pour des raisons humanitaires fondées notamment sur des motifs familiaux. L'alinéa 3 dispose que si le demandeur d'asile est un mineur non accompagné et qu'un ou plusieurs membres de sa famille se trouvant dans un autre Etat membre peuvent s'occuper de lui, les Etats membres réunissent si possible le mineur et le ou les membres de sa famille.

${ }^{36}$ Article 6-1: «si le demandeur d'asile est un mineur non accompagné, l'Etat membre responsable de l'examen de la demande est celui dans lequel un membre de sa famille se trouve légalement pour autant que ce soit dans l'intérêt du mineur».

${ }^{37}$ Ce critère repose sur l'idée que le traitement des demandes d'asile des différents membres d'une famille par un seul et même Etat est une mesure de nature à permettre un examen approfondi des demandes et la cohérence des décisions prises à leur égard. Il faut noter que le Conseil, contrairement à la Commission, n'a pas exclu du bénéfice de cette disposition les demandeurs d'asile se trouvant hors du cadre d'une procédure normale (procédure accélérée par exemple). La disposition finale évite par ce biais l'apparition de discriminations entre différentes catégories de demandeurs d'asile. 
Ces dispositions sont un progrès certain ${ }^{38}$ mais la générosité du règlement Dublin à l'égard des familles ne doit pas faire illusion. En dépit des apparences, les nouvelles dispositions sur la famille sont une concession faite aux voix critiques de la Convention de 1990 mais elle ne changent pas la philosophie d'attribution de la responsabilité. Les articles consacrés au regroupement familial sont pour la plupart une codification des décisions du Comité de l'article $18^{39}$. Et surtout, la protection des familles semble accessoire, comme le montre le préambule du règlement (point 6) : «il y a lieu de préserver l'unité des familles dans la mesure où ceci est compatible ${ }^{40}$ avec les autres objectifs poursuivis par l'établissement de critères et mécanismes de détermination de l'Etat responsable de l'examen d'une demande d'asile ». Dans sa proposition, la Commission considérait également que l'article 8 ne pourrait utilement s'appliquer que s'il ne portait pas préjudice à d'autres objectifs importants des instruments de la politique communautaire en matière d'asile, " notamment à l'objectif d'un traitement rapide des demandes, en particulier des demandes manifestement infondées $\gg{ }^{41}$. A aucun moment la garantie de l'unité familiale n'est donc conçue comme susceptible de fonder le nouveau système de désignation de l'Etat responsable. Le contrôle des flux migratoires reste l'axe central du mécanisme Dublin.

38 La Convention de Dublin ne prenait en compte la situation des familles que très partiellement. Elle garantissait seulement que (article 4) «si le demandeur d'asile a un membre de sa famille qui s'est vu reconnaître la qualité de réfugié au sens de la convention de Genève dans un Etat membre et qui y réside légalement, cet Etat est responsable de l'examen de la demande, à la condition que les intéressés le souhaitent. Le membre de la famille concerné ne peut être que le conjoint du demandeur d'asile ou son enfant mineur célibataire de moins de 18 ans, ou son père ou sa mère si le demandeur d'asile est lui-même un enfant mineur célibataire de moins de 18 ans ». Il arrivait fréquemment que plusieurs Etats membres soient déclarés compétents pour les membres d'une même famille. La seule possibilité de « réunir » ces familles était alors l'application de la clause humanitaire (article 9) qui permet à un Etat non responsable d'assumer la responsabilité sur divers motifs notamment familiaux. Mais, d'une part, l'Etat sollicité n'était pas obligé d'accepter le transfert de responsabilité. D'autre part, l'article 9 ne conférait pas un droit individuel au profit du demandeur d'asile lui permettant de voir sa demande examinée par un Etat donné. Enfin, la notion de membre de la famille était entendue très restrictivement. Souvent donc la clause humanitaire n'était pas activée. La seule pour assurer l'unité familiale était le recours à la clause de souveraineté. Cette clause de l'article 3-4 de la Convention permet à un Etat de décider d'examiner une demande d'asile, avec le consentement du demandeur, même s'il n'est pas compétent au titre des critères Dublin.

${ }^{39}$ Ainsi par exemple, l'article 6-1 correspond à l'un des cas visés dans la décision 1/2000 du comité de l'article 18. De même, l'article 8 vise à régler par un critère autonome et contraignant certains des cas visés par la décision 1/2000. Enfin, les critères choisis par l'article 14 permettent de donner force obligatoire à certain des cas de figure envisagés dans la décision 1/2000 sous forme de « lignes directrices » sujettes à une appréciation au cas par cas.

40 c'est nous qui soulignons.

${ }^{41}$ Proposition de règlement, commentaire de l'article 8. 
On note d'ailleurs que Dublin 2 est conçu en relation avec la norme communautaire sur le regroupement familial ${ }^{42}$. Le règlement Dublin en effet a été modelé en fonction des termes de la proposition de directive sur le regroupement familial des ressortissants d'Etats tiers ${ }^{43}$. Cette adaptation est manifeste, tout d'abord, dans la définition des membres de la famille (article 2, i). Selon le règlement, est membre de la famille le conjoint, ou son partenaire non marié, engagé dans une relation stable, lorsque la législation ou la pratique de l'Etat membre réserve aux couples non mariés un traitement comparable aux couples mariés en vertu de sa législation sur les étrangers. Sont également membres de la famille les enfants mineurs des couples mariés ou non mariés s'ils sont célibataires et sont à charge. Enfin, sont membres de la famille le père, la mère ou le tuteur lorsque le demandeur d'asile ou le réfugié est mineur et non marié. Cette définition de la famille correspond à celle retenue par la (proposition de) directive sur le regroupement familial.

De plus le règlement est plus restrictif que la proposition de règlement, qui n'imposait l'existence de constitution de la famille dans le pays d'origine que pour les couples non mariés. Il est aussi plus strict que la proposition de directive sur le regroupement familial : cette dernière prévoyait à son article 9 que les Etats membres peuvent limiter l'application des dispositions de ce chapitre aux réfugiés dont les liens familiaux sont antérieurs à la reconnaissance de ce statut alors que le règlement impose cette limitation. Il faut que soit d'abord démontré que la famille existait déjà dans le pays d'origine. La rigueur $\mathrm{du}$ règlement Dublin n'est pas fortuite. Elle illustre la crainte que de «faux réfugiés» se servent du mécanisme Dublin pour contourner le (futur) dispositif sur le regroupement familial ${ }^{44}$. Peut se comprendre de la même manière l'abandon de la disposition au titre de laquelle était membre de la famille la personne «avec laquelle existe un lien de parenté et qui vivait dans le même foyer dans le pays d'origine si l'une des personnes concernées est dépendante de l'autre » (art $2 \mathrm{i}$ de la proposition de règlement) ${ }^{45}$. Cette définition était souple et favorable au regroupement familial des réfugiés. Le règlement au contraire

${ }^{42}$ COM(1999)638 final - 1999/0258. Ainsi la Commission considérait dans son document «Réexamen de la Convention de Dublin », §46 que la Communauté mettant actuellement en place les règles générales sur le regroupement familial, il est important d'assurer que l'acte déterminant la responsabilité pour l'examen de la demande d'asile soit en cohérence avec la future législation communautaire sur le regroupement familial.

43 Devenue depuis : Directive 2003/86 du Conseil du 22 septembre 2003 relative au regroupement familial, J.O. L251, 3.10.2003, p. 12-18.

${ }^{44} \mathrm{La}$ Commission dans sa proposition s'est en effet inquiétée du fait que «ces dispositions (sur l'unité familiale) puissent être détournées de leur finalité pour contourner les règles relatives au regroupement familial proposées par la Commission dans sa proposition de directive du Conseil relative au regroupement familial. »

${ }^{45}$ Le HCR soutenait cette proposition, conforme à l'approche défendue par le Comité Exécutif dans ses conclusions N88 (XLX) de 1999. 
choisit une définition stricte, conforme aux termes de la proposition de directive regroupement familial. Enfin, la peur du contournement des dispositions sur l'immigration explique la limitation du champ d'application de l'article $7 \mathrm{du}$ règlement. Cet article dispose que si un membre de la famille du demandeur d'asile a été amis à résider en tant que réfugié dans un Etat membre, cet Etat est responsable de l'examen de la demande. Il ne prévoit donc la réunification qu'avec une personne bénéficiant du statut de réfugié ${ }^{46}$, alors que le $\mathrm{HCR}^{47}$ et diverses ONG recommandaient de garantir cette unité familiale avec un membre de la famille sous protection subsidiaire ou simplement légalement résident dans l'Etat membre. Une telle possibilité assurerait en effet une meilleure intégration du demandeur d'asile dans la société d'accueil, ainsi qu'une certaine stabilité, et diminuerait par ailleurs les risques de migrations secondaires. L'explication la plus plausible de ces différents choix normatifs semble donc être la crainte de voir des ressortissants d'Etats tiers ne répondant pas à la définition de membre de la famille (au sens de la proposition de directive) utiliser le regroupement familial des réfugiés pour accéder à l'UE.

Bien qu'il ait été adopté six mois avant la directive sur le regroupement familial des ressortissants d'Etats tiers ${ }^{48}$, le règlement 343/2003 est donc modelé en fonction des objectifs des Etats en matière de réunification familiale. Le système Dublin n'ouvre pas de voie permettant de contourner les règles générales sur l'immigration. De même, les Etats subordonnent la réforme réelle des mécanismes Dublin à l'adoption des normes communautaires harmonisant les législations nationales sur l'asile. La réforme est donc différée : elle devra être postérieure à l'harmonisation.

§2-Une réforme différée à la période post-harmonisation

La Convention de 1990 désignait l'Etat responsable d'une demande d'asile sur la base de six critères, prédéfinis, contraignants et qui pour l'essentiel lient la responsabilité au rôle joué par l'Etat $^{49}$. Souhaitant réformer ces critères, la

\footnotetext{
${ }^{46}$ Voir les commentaires de ECRE, précités, p. 8; Amnesty International, précité, p. 5; analyses du comité économique et social, précitées.

${ }^{47}$ Voir HCR Observations on the European Commission's proposal for a Council Regulation establishing the criteria and mechanisms for determining the Member State responsible for examining an asylum application lodged in one of the Member States by a third-country national, p. 3.

${ }^{48}$ Directive 2003/86 du Conseil du 22 septembre 2003 relative au regroupement familial, J.O. L251, 3.10.2003, p. 12-18.

${ }^{49}$ Ainsi, l'Etat est désigné compétent parce qu'il a dans le passé décidé d'octroyer le statut de réfugié à un membre de la famille du demandeur d'asile (critère 1), ou parce qu'il a délivré un permis de séjour (critère 2) ou un visa (critère 3), ou parce qu'il n'a pas empêché l'entrée illégale (critère 4) ou parce qu'il a autorisé l'entrée (critère 5). Ce n'est que lorsque aucun de ces critères ne peut être déclenché que l'on déterminera la responsabilité sur la base d'un
} 
Commission a considéré différentes solutions de remplacement ${ }^{50}$. Elle a envisagé en premier lieu un modèle qui attribuerait la responsabilité au dernier Etat de transit connu dans l'UE. Ce système réduirait les problèmes de preuve car s'il est généralement difficile de savoir par quel Etat un demandeur d'asile est entré dans l'UE, il est plus aisé d'identifier le dernier Etat de transit. Toutefois, le projet a été abandonné car il pénaliserait les Etats pour l'abolition de leurs frontières internes. La Commission a ensuite suggéré un système radical au titre duquel tous les demandeurs d'asile d'une même nationalité seraient affectés à un seul et même Etat membre. Cette approche présente l'avantage de tenir compte, pour le rattachement des demandeurs d'asile aux Etats, de l'existence de liens culturels ou historiques entre certains Etats membres et certaines nationalités. Mais elle repose sur une attribution relativement arbitraire des demandeurs d'asile à un Etat et serait, en cas de crise grave dans un pays et de mouvements de populations importants, en contradiction avec l'objectif de partage équitable des demandeurs d'asile entre les Etats membres.

La Commission a ensuite écarté chacune de ces possibilités et conclu que l'alternative la plus crédible au système de 1990 est celle qui ferait dépendre la responsabilité de l'examen de la demande d'asile exclusivement du lieu où la demande a été présentée. Seule cette logique permettrait de «mettre en place un système clair et viable répondant à un certain nombre d'objectifs : rapidité et certitude, éviter les réfugiés en orbite, traitement du problème des demandes d'asile multiples et garanties de l'unité familiale $)^{51}$. Malgré les arguments favorables au changement, le Conseil a préféré le statu quo. Le Conseil n'a pas choisi de consacrer l'alternative au système de 1990, qui consistait à désigner responsable l'Etat dans lequel le demandeur d'asile dépose sa demande. Le refus du changement est significatif de la posture choisie par les Etats : ils ont refusé de consacrer un système de laissez faire et préféré maintenir un mécanisme qui tente de contrôler les trajectoires des migrants d'asile et leur répartition entre les Etats de 1'Union.

Ce choix est regrettable parce qu'il repose sur une vision contestable de l'asile. Les institutions communautaires assimilent le choix d'un Etat de destination à une stratégie d'abus des procédures d'asile. Ainsi, la Commission indiquait la nécessité de lutter «contre le détournement et l'abus des procédures d'asile, en empêchant le demandeur d'asile de choisir l'Etat dans lequel il va demander

critère qui échappe à l'Etat : le lieu de dépôt de la demande d'asile, autrement dit, le choix du demandeur d'asile.

${ }^{50}$ Voir Réexamen de la Convention de Dublin, précité.

${ }^{51}$ Proposition de règlement du Conseil établissant les critères et mécanismes de détermination de l'Etat membre responsable de l'examen d'une demande d'asile présentée dans l'un des Etats membres par un ressortissant d'un pays tiers, précitée, exposé des motifs. 
l'asile» ${ }^{52}$. Ces propos révèlent la méfiance à l'égard de ce que l'on est désormais convenu d'appeler l'asylum shopping. Au sens strict et neutre, l'asylum shopping est le processus par lequel un demandeur d'asile compare plusieurs pays de possible destination et sélectionne sur la base de différents critères celui dans lequel il va finalement demander l'asile. Mais les institutions communautaires emploient ce terme dans un autre sens, décrivant le dépôt de demandes d'asile multiples simultanément dans le but de contourner les systèmes nationaux d'asile. ${ }^{53} \mathrm{La}$ Commission a ainsi progressivement établi une différence entre le fait de choisir un Etat de destination sur la base de critères familiaux, culturels ou linguistique, (stratégie considérée comme légitime), et la sélection d'un Etat en raison du droit applicable (sélection considérée illégitime $)^{54}$. Cette distinction est inadéquate car elle ne prend pas en considération l'importance que peut revêtir, pour un demandeur d'asile, la différence d'interprétation de la Convention de Genève, l'accès possible à une protection subsidiaire, ou encore la définition d'un Etat sûr ${ }^{55}$.

Une autre raison justifie le refus de laisser les demandeurs d'asile choisir leur Etat de destination. Pour la Commission ${ }^{56}$, cette alternative nécessiterait une harmonisation dans d'autres domaines tels que la procédure d'asile, les conditions d'accueil, l'interprétation de la définition du terme "réfugié » et la protection subsidiaire pour réduire les facteurs qui inciteraient éventuellement les demandeurs d'asile à choisir entre les Etats membres au moment où ils introduisent leur demande. En effet, au stade actuel de l'édification du régime d'asile européen commun, il existe entre les Etats membres, dans les procédures d'admission au statut de réfugié, les conditions d'accueil des demandeurs d'asile et l'organisation des formes complémentaires de protection, «des différences

\footnotetext{
${ }^{52}$ Réexamen de la Convention de Dublin, précité, $\$ 40$.

53 Comme l'atteste par exemple l'argumentaire sur les réseaux de passeurs qui, selon la Commission, inciteraient les migrants économiques à se rendre dans certains Etats membres et à y demander l'asile pour éviter l'expulsion. In Réexamen de la Convention de Dublin, précité, $\$ 42$.

${ }^{54}$ Voir exposé des motifs des diféfrentes propositions de directive d'harmonisation.

55 En fait, Commission et Conseil consacrent l'idée selon laquelle les demandeurs d'asile doivent demander l'asile dans le premier Etat sûr qu'ils réussissent à atteindre. Cette approche, bien que désormais acceptée par tous les Etats membres de l'UE qui ont intégré le concept d'Etat tiers sûr dans leur système juridique, ne trouve aucun fondement juridique dans la Convention de Genève et n'est pas partagée par l'ensemble des Etats de la Communauté internationale. Il n'est donc pas surprenant qu'elle ne s'accorde pas avec la décision 15 du comité exécutif du UNHCR (Conclusion N¹5 du Comité exécutif du HCR, (No. 15 (XXX) - 1979) Réfugiés sans Etat d'asile), décision dans laquelle le HCR recommande de prendre en considération autant que possible les intentions des demandeurs d'asile quand à l'Etat dans lequel ils désirent demander l'asile.

${ }^{56}$ Réexamen de la Convention de Dublin, précité $§ 59$.
} 
susceptibles d'avoir une influence sur l'orientation des flux de demandeurs d'asile. ${ }^{57}$

La Commission craint donc que les Etats membres offrant le niveau de protection le plus élevé de l'UE soient le réceptacle de l'ensemble des demandeurs d'asile et elle propose de différer la réforme. Cette crainte apparemment partagée par le Conseil - repose sur le postulat qu'un changement de méthode de détermination de l'Etat compétent, laissant aux demandeurs d'asile le choix de leur Etat de destination, équivaudrait à abandonner la sécurité offerte par un système contrôlé et dirigiste et à accepter les conséquences d'un système libéral, de distribution spontanée des réfugiés entre les Etats membres. Un tel raisonnement est contestable car il suppose, a contrario, qu'il est possible de contrôler effectivement la répartition des demandeurs d'asile en Europe. Cela revient à nourrir la fiction selon laquelle les Etats ont la capacité d'empêcher un demandeur d'asile de se rendre dans son Etat de prédilection. L'expérience a pourtant montré que, en usant toutes voies légales possibles ou au prix de la clandestinité, les demandeurs d'asile parviennent à échapper à l'application des critères Dublin.

De surcroît, pour refuser le choix de l'Etat de destination, les institutions communautaires arguent de la situation actuelle -et temporaire - de nonharmonisation des droits nationaux. Selon la Commission, c'est la diversité des droits nationaux qui suscite les stratégies de comparaison entre les Etats et conduit à privilégier les Etats les plus protecteurs. C'est pourquoi elle propose de reporter la mise en place du système de libre choix à l'après harmonisation. Ce raisonnement n'emporte pas non plus la conviction car rien ne garantit que l'harmonisation des droits nationaux d'asile permettra d'équilibrer la répartition des demandeurs d'asile entre les Etats. En effet, pour choisir leur Etat d'accueil, les demandeurs d'asile prennent en compte une multiplicité de critères ${ }^{58}$ et, s'ils comparent les droits garantis (protection contre le refoulement, sécurité sociale, possibilité de travailler etc.), ils prennent également en considération la présence de communautés nationales ou linguistiques, de réseaux d'entraide, la proximité culturelle avec l'Etat membre etc. Aussi, l'harmonisation n'aura aucune incidence sur le choix d'un Etat fondé sur des critères non juridiques. Par ailleurs, dans la mesure où le traité ne prévoit que des directives

\footnotetext{
${ }^{57}$ Proposition de règlement du Conseil établissant les critères et mécanismes de détermination de l'Etat membre responsable de l'examen d'une demande d'asile présentée dans l'un des Etats membres par un ressortissant d'un pays tiers précitée.

${ }^{58}$ Pour une étude détaillé de ce que l'on a appelé les "push and pull factors" (ie.. les facteurs incitant les demandeurs d'asile à quitter leur pays ou à choisir certaines destination en particulier), voir A. Bocker et T. Havinga, Asylum migration to the European Union : patterns of origin and destination, Institute for the Sociology of law, Nijmegen, étude publiée par l'Office des Publications des Communautés Européennes, 1998.
} 
d'harmonisation partielle, les droits nationaux sur l'asile, même dans les domaines couverts par l'harmonisation, ne seront pas unifiés. Par conséquent, l'harmonisation ne mettra pas fin aux tactiques d'asylum shopping fondées sur les écarts de niveaux de protection offerts par les différents Etats membres. ${ }^{59}$

Reposant sur des postulats erronés, le règlement choisit donc des solutions dont on a toutes les raisons de penser qu'elles sont inadaptés aux réalités de l'asile dans l'Union européenne et compromettront probablement l'application effective du "nouveau" système Dublin. Il n'est guère besoin de pessimisme pour faire la chronique des dysfonctionnements prévisibles d'un système non réformé dans un système non harmonisé. Le système Dublin 2 se prive des atouts de la méthode envisagée par la Commission. Un système désignant responsable l'Etat dans lequel le demandeur d'asile dépose sa demande d'asile limiterait en effet les stratégies de recours à la clandestinité dans la mesure où les individus n'auraient pas à contourner le dispositif Dublin pour pouvoir choisir leur Etat de destination. Cela contribuerait par effet ricochet à lutter contre les réseaux de passeurs clandestins qui exploitent les demandeurs d'asile. Par ailleurs, les demandeurs d'asile ne cherchant plus à éviter un mécanisme qu'ils considèrent injustes, seraient moins incités à détruire leurs documents d'identité ou à mentir sur leur route européenne, ce qui réduirait les problèmes de preuves qui ont miné la bonne application du système Dublin. La stratégie de demande d'asile multiple devrait également logiquement disparaître, ce qui soulagerait les systèmes nationaux d'asile. Enfin, l'unité familiale pourrait être assurée plus spontanément.

Au contraire, le statu quo laisse ouvertes à peu près toutes les stratégies par lesquelles les demandeurs d'asile rejoignent leur Etat de prédilection ${ }^{60}$. Les

\footnotetext{
${ }^{59}$ La Commission reconnaît d'ailleurs que les différences susceptibles d'avoir une incidence sur les orientations des demandeurs d'asile subsisteront, bien que sous une forme atténuée, après que les directives proposées par la Commission sur ces matières seront entrées en vigueur, in Proposition de règlement du Conseil, précitée.

${ }^{60}$ Il n'est pas impossible que le règlement ouvre même la voie à de nouvelles «stratégies » qui mettront à mal l'efficacité du dispositif Dublin 2. Le nouvel article 10-1 attribue la responsabilité à l'Etat par lequel le demandeur d'asile est entré irrégulièrement dans l'UE. Mais cette responsabilité prend fin douze mois après la date du franchissement irrégulier de la frontière. Au titre de l'alinéa 2, si les circonstances de l'entrée ne peuvent être établies et que le demandeur d'asile entré irrégulièrement en Europe a séjourné dans un Etat membre pendant une période continue d'au moins cinq mois avant l'introduction de sa demande, cet Etat membre est responsable de l'examen de la demande d'asile. On peut imaginer qu'un demandeur d'asile qui veut demander l'asile dans un Etat de l'Union mais ne peut s'y rendre qu'en passant par d'autres Etats membres, a plus que jamais intérêt à entrer clandestinement en Europe, à se rendre clandestinement dans son Etat de destination, à y rester sans se déclarer pendant 5 mois (ou 12 mois pour avoir toute garantie, en application de l'alinéa 1), puis à y déposer une demande d'asile. Par application de l'article 10, il pourra voir sa demande examinée par l'Etat de son choix.
} 
projets d'articles 4-3 et 20-2 ayant été abandonnées, le nouveau règlement ne mettra pas un terme aux stratégies d'évitement du dispositif de désignation de l'Etat responsable : un demandeur d'asile voulant échapper à un transfert pourra toujours le retarder ou y échapper en retirant sa demande de statut de réfugié. En outre, les nombreux recours observés contre l'application du système Dublin 1 démontrent que lorsque les demandeurs d'asile ne recourent pas à des moyens hasardeux ou illégaux pour éviter les critères Dublin, ils tentent toutes les stratégies possibles de contestation par la voie légale. Le succès de certains recours (en particulier dans certains Etats) contre le transfert d'un Etat membre à un autre tient à ce que certaines juridictions sont réceptives aux arguments des demandeurs d'asile et remettent explicitement en question le niveau de protection conféré aux réfugiés dans d'autres Etats membres ${ }^{61}$. Le règlement Dublin ne donnant aucune raison à ces juges de renoncer à leur jurisprudence, on comprend qu'en attendant l'harmonisation, l'effectivité du dispositif Dublin 2 pourrait relever du vœu pieux.

Cette observation conduit donc à questionner l'ordre d'adoption des normes sur l'asile prévu par le traité CE et notamment la décision d'adopter le mécanisme Dublin lors de la première vague de normes réalisant la politique communautaire d'asile (normes devant être adoptées pour 2004). Il est permis de penser que l'adoption du mécanisme Dublin aurait dû avoir lieu à la fin du processus d'harmonisation des droits d'asile plutôt qu'au début. Un des membres de la Commission, M. Fortescue fait d'ailleurs le pronostic suivant: " quand toutes les pièces seront mises en place, et lorsque nous aurons vraiment initié un véritable système d'asile commun, ce sera le moment de réexaminer Dublin $2 .{ }^{62}$ » Peut on mieux dire que Dublin 2 est conçu comme un système temporaire, destiné à être remanié assez rapidement et dont les nombreuses imperfections sont explicables par les contraintes tenant au moment de son adoption et à son antériorité par rapport aux autres normes prévues par le titre IV

${ }^{61}$ On connaît en effet la jurisprudence spectaculaire de la Chambre des Lords en décembre 2000. La Cour britannique, ayant constaté les divergences d'interprétation de la Convention de Genève et relevé qu'en droit britannique, à la différence de la France et l'Allemagne, la qualité de réfugié peut être reconnue aux personnes persécutée par des acteurs non étatiques, a décidé de ne pas procéder au transfert prévu. Elle a considéré que l'existence de différentes interprétations de la Convention de Genève impose au Royaume Uni de considérer en substance une demande d'asile, au titre de la dérogation humanitaire, et de renoncer au transfert du demandeur d'asile vers l'Etat compétent au titre des critères Dublin. Voir Elspeth Guild, Case-Law on the Dublin Convention in the United Kingdom, in Claudia Faria (ed.) The Dublin Convention on Asylum, between Reality and Aspirations, Institut Européen d'Administration Publique, 2001.

${ }^{62}$ «I do not at all rule out that when all the building blocks are in place and when we move towards something which is a genuine common asylum system, there could well be a case for revisiting Dublin II » cité in Nineteenth Report of the UK Parliament, 19 March 2002, by the Select Commitee appointed to consider Union documents and other matters relating to the European Union, www.parliament-the-stationery-office.co.uk, point 35 (traduit par nos soins). 
TCE ? L'efficacité du système Dublin 2 semble donc reposer sur les quelques nouvelles dispositions qui tentent de rationaliser et de perfectionner la coopération entre les Etats. En réalité, l'absence de réforme substantielle trahit la volonté des Etats de confier l'efficacité du système Dublin au dispositif Eurodac.

\section{Section 2. L'EFFICACITE DELEGUEE}

N'étant pas substantiellement réformé et se déployant dans un espace où les droits de l'asile ne sont pas harmonisés, Dublin 2 a toutes les chances de ne pas être effectif. Les espoirs de son bon fonctionnement reposent en effet uniquement sur quelques dispositions ajustant les mécanismes de 1990 et s'efforçant de faciliter la coopération inter étatique (1). Il semble que les Etats aient placé en EURODAC leur espoir de voir le système Dublin fonctionner (2).

\section{§1- Quelques ajustements techniques pour une effectivité incertaine}

Le toilettage du dispositif Dublin concerne tout d'abord les délais, une réforme ayant été souhaitée pour accélérer la détermination de l'Etat compétent et l'organisation du transfert ${ }^{63}$. Le texte de 1990 permettait en effet une très longue procédure puisque trois délais venaient s'ajouter. Un Etat membre disposait d'un premier délai de 6 mois (à compter du dépôt de la demande d'asile) pour demander à un autre Etat membre de prendre en charge un demandeur d'asile ${ }^{64}$. L'Etat requis disposait alors d'un délai de 3 mois pour répondre ${ }^{65}$. Si l'Etat requis acceptait la responsabilité, le transfert du demandeur d'asile était organisé et devait, selon l'article 11-5, intervenir au plus tard un mois après l'acceptation de la demande de prise en charge ou un mois après l'issue de la procédure contentieuse éventuellement engagée par l'étranger contre la décision de transfert si la procédure était suspensive ${ }^{66}$. L'ensemble de la procédure de détermination de l'Etat responsable, et le cas échéant du transfert, pouvait donc prendre 9 mois, ce qui était problématique à la fois pour les Etats et pour les demandeurs d'asile, placés dans une situation de grande incertitude ${ }^{67}$. Le délai

\footnotetext{
${ }^{63}$ Voir Friedrich Löper, The Dublin Convention Provisions on Time Limits and the Exchange of Informations: Which Are the Provisions and What are the Problems, in Claudia Faria (ed) The Dublin Convention on Asylum, Between Reality and Aspirations, Institut Européen d'Administration Publique, 2001, p. 7

${ }^{64}$ Article 11-1.

${ }^{65}$ Article 11-4

${ }^{66}$ La Convention de Dublin réglait aussi les hypothèses de reprise en charge des demandeurs d'asile. Selon la Convention, l'Etat requis pour une reprise en charge est tenu de répondre à la demande dans un délai de huit jours à compter de la saisine.

${ }^{67}$ De plus, le délai de demande de prise en charge est critiquable car il contredit le principe énoncé dans le préambule de la Convention de Dublin selon lequel la demande d'asile devrait être examinée promptement. Il est également en conflit avec le délai préconisé par la
} 
de réponse à la demande de prise en charge fut également été considéré trop long $^{68}$. En revanche le délai pour procéder au transfert fut considéré trop bref car le transfert est une procédure complexe dans laquelle interviennent plusieurs autorités et qui peut être entravée par divers événements, comme la maladie du demandeur d'asile ou sa disparition.

Le règlement tente d'améliorer le dispositif en modifiant les délais applicables à chaque phase de la procédure. Désormais les Etats ont 3 mois ${ }^{69}$ (65 jours ouvrables selon la proposition) pour demander à un autre Etat de prendre en charge un demandeur d'asile. L'Etat membre requis doit statuer sur la requête aux fins de prise en charge dans un délai de deux mois (la proposition prévoyait un mois). L'absence de réponse dans ce délai équivaut à acceptation de la requête et entraîne obligation de prendre en charge la personne concernée (article 18$)^{70}$. Cette disposition, bien sûr, est un mécanisme incitatif : les Etats doivent être prompts dans leur réponse afin d'assurer le bon fonctionnement du système de détermination de la responsabilité-transfert. Enfin, le transfert est réalisé dès que cela est matériellement possible et, au plus tard dans un délai de six mois à compter de l'acceptation de la demande de prise en charge (article 193). Cependant, au titre de l'article 19-4 (article que la proposition n'avait pas envisagé), si le transfert n'est pas exécuté dans le délai de 6 mois, la responsabilité incombe à l'Etat membre auprès duquel la demande d'asile a été introduite. Ici encore, le règlement incitent les Etats à la diligence. Toutefois, ce délai peut être porté à un an au maximum s'il n'a pas pu être procédé au transfert en raison d'un emprisonnement du demandeur d'asile ou à dix-huit mois au maximum si le demandeur d'asile prend la fuite. Ces nouveaux délais doivent être accueillis positivement car ils tiennent compte de la difficulté rencontrée par les Etats. Cependant on ne doit pas surestimer l'importance de la réforme car le délai total pour l'organisation du transfert reste de 11 mois dans l'hypothèse basse, alors qu'il était de 9 mois dans le dispositif d'origine.

Pour assurer l'efficacité du dispositif, le règlement procède également à quelques amendements qui vont «huiler» la coopération inter étatique. Il programme l'adoption de deux listes qui préciseront les éléments de preuve et

proposition de directive sur les normes minimales en matière de procédure. L'article 18 de cette proposition dispose qu'un Etat peut déclarer inadmissible une demande d'asile, si un autre Etat membre est responsable de l'examen de la demande. L'article 23 accorde 65 jours ouvrables pour la prise de décision.

${ }^{68}$ Les Etats s'étaient d'ailleurs, dans la décision 1/97 (article 4) engagés à mettre tout en œuvre pour répondre dans un délai d'un mois.

${ }^{69}$ Article $17 \mathrm{du}$ règlement.

${ }^{70}$ Cette disposition n'est pas à l'abri de toute critique car il se peut que ce soit le demandeur d'asile, et non l'Etat qui soit « pénalisé » par cette absence de réponse. 
les indices permettant d'établir la responsabilité ${ }^{71}$. Il contient aussi des dispositions organisant les échanges d'informations entre les Etats membres (article 21). Enfin le règlement prévoit que les Etats membres peuvent établir entre eux, sur une base bilatérale, des arrangements administratifs pour accroître l'efficacité du dispositif Dublin. Ces arrangements prévus par l'article 23 peuvent, d'une part porter sur des échanges d'officiers de liaison, et d'autre part sur une simplification des procédures et un raccourcissement des délais applicables à la transmission et à l'examen des requêtes de prise en charge ou de reprise en charge.

\section{§2. EURODAC ou les espoirs du Conseil.}

L'impression générale qui se dégage à la lecture des deux règlements et que ces quelques aménagements ne suffiront pas à assurer l'efficacité de Dublin 2. Il semble même que le Conseil en était conscient et a implicitement confié le soin d'assurer l'efficacité de Dublin 2 au système Eurodac, la base de données d'empreintes digitales des demandeurs d'asile mise sur pied en $2000^{72}$. Le préambule du règlement 343/2003 indique ainsi que (point 11) "l'exploitation du système Eurodac rendra plus aisée la mise en œuvre du présent règlement."

Eurodac fut en effet conçue pour aider les Etats à identifier le pays responsable de l'examen d'une demande d'asile et depuis le 15 janvier 2003, tous les demandeurs d'asile voient leurs empreintes digitales relevées lorsqu'ils présentent une demande d'asile dans le cadre de la procédure normale de demande d'asile. Les empreintes sont ensuite envoyées au point national d'accès de chacun des Etats participant et transmises sous forme numérique à une unité centrale à Bruxelles. Elles sont alors automatiquement comparées aux empreintes de toutes les personnes qui, depuis le 15 janvier 2003, ont introduit une demande d'asile dans l'UE ainsi qu'avec les empreintes des personnes qui ont été appréhendées alors qu'elles tentaient d'entrer illégalement dans l'union européenne mais à qui l'entrée n'a pas pu être refusée. Si les empreintes digitales figurent dans Eurodac, l'information est communiquée au pays ayant transmis les empreintes. Celui ci pourra alors envoyer le demandeur dans le pays où il a présenté sa première demande ou par lequel il est entré dans l'Union européenne afin que ce deuxième pays décide de la suite à donner à la demande.

\footnotetext{
${ }^{71}$ Article 18. Les éléments de preuve permettent d'établir la preuve de la responsabilité aussi longtemps qu'elle n'est pas réfutée par une preuve contraire. Les indices sont des éléments indicatifs qui, tout en étant réfutables, peuvent être suffisants dans certains cas, en fonction de la preuve probante. C'est le comité de l'article 27, constitué de représentants d'Etats membres qui établira ces listes de preuve et indices.

${ }^{72}$ Règlement du Conseil CE 2725/2000 du 11 décembre 2000 concernant la création du système Eurodac pour la comparaison des empreintes digitales aux fins de l'application efficace de la Convention de Dublin, JOCE L 316 du 15.12.2000, p. 1.
} 
Eurodac est donc appelé à jouer un rôle dans le fonctionnement du système Dublin, notamment dans le système de preuve et transmission des informations entre les Etats. Ainsi par exemple, au titre du règlement de septembre 2003, lorsqu'une requête de reprise en charge est fondée sur des données fournies par l'unité centrale d'Eurodac (et vérifiées par l'Etat requérant), l'Etat membre requis reconnaît sa responsabilité. La cessation de la responsabilité ne peut être invoquée que sur la base d'éléments de preuve matériels ou de déclarations circonstanciées et vérifiables du demandeur d'asile.

Ayant renoncé à réformer substantiellement le système Dublin, les Etats semblent donc s'en remettre à Eurodac pour déceler les dépôts multiples de demandes d'asile et supprimer certaines difficultés de preuve, notamment quant au lieu d'entrée en Europe. Cette large confiance placée dans le système Eurodac est un pari et un symbole. Elle illustre la tendance "défensive » du système Dublin qui repose sur l'illusion du contrôle et associe la responsabilité de l'examen des demandes d'asile au contrôle des frontières. On ne saurait en effet omettre que, si le système Eurodac peut aider à déterminer quel Etat est responsable de l'examen de la demande d'asile, il fut avant tout conçu pour déterminer qui est entré illégalement dans l'Union européenne.

\section{Conclusion :}

Le système Dublin mis en place en 1990 a mal fonctionné. Dans ses travaux préparatoires, la Commission proposait une réforme radicale du système qui rénovait l'esprit du système Dublin et pouvait en assurer l'efficacité. Le conseil au contraire a choisi le statu quo, l'attente et la réforme annoncée n'a pas eu lieu. La logique d'attribution de la responsabilité est reconduite et la caractéristique du règlement du 18 février 2003 semble être une certaine myopie à l'égard des migrations d'asile. Les institutions communautaires oscillent entre une approche méfiante des stratégies de déplacement et de choix de l'Etat de destination, l'utopie d'un possible contrôle de ces déplacements et de ces choix, et l'illusion que Dublin 2 pourrait être efficace. L'impression qui domine à la lecture des règlements de février et septembre 2003 est que la réforme de la Convention de Dublin a été manquée. Il reste à déterminer si c'est la situation de dépendance à l'égard du processus d'harmonisation qui explique ce demi-échec, auquel cas il conviendra d'attendre la réforme du système Dublin 2. Ou peut être faut-il plus généralement se demander si la conception même d'un instrument qui affecte ex ante et strictement un Etat à un individu est une approche adéquate des réalités migratoires de l'asile en Europe. 